\title{
Société suisse de chirurgie maxillo-faciale (SSCMF)
}

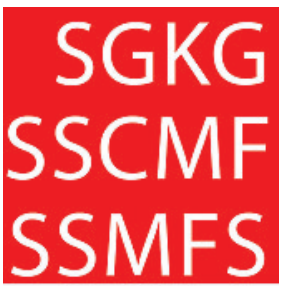

WWW.SGKG.ORG
Correspondance:

Prof. Hans-Florian Zeilhofer, dr en médecine humaine et dentaire, $\mathrm{dr}$ honoris causa Clinique de chirurgie maxillo-faciale

Universitätsspital Basel

Spitalstr. 21

CH-4031 Bâle

Tél. 0612657344

hfzeilhofer@uhbs.ch
La chirurgie maxillo-faciale est une discipline relativement jeune qui s'est développée suite aux expériences faites au cours des deux guerres mondiales dans le traitement de graves blessures crâniennes par balle. A l'époque, chirurgiens et dentistes constatèrent qu'ils ne pouvaient pas mener à bien les reconstructions complexes du crâne facial et rétablir les fonctions de mastication, de langage, de déglutition et de respiration des patients en traumatologie sans bénéficier des connaissances de l'autre. C'est pourquoi la chirurgie maxillo-faciale se fonde aujourd'hui sur une double formation en médecine humaine et dentaire.

\section{Une discipline à double qualification}

En vue de l'octroi du titre de spécialiste, la Suisse, comme de nombreux autres pays européens, exige une double qualification en médecine humaine et en médecine dentaire. La personne qui opte pour cette formation a donc de longues années d'études devant elle si l'on considère qu'il faut compter 10 ans d'études et 6 ans de formation postgraduée pour l'obtention du titre.

\section{Développement de la société en Suisse}

Hugo Obwegeser à Zurich et Bernd Spiessl à Bâle ont grandement contribué au développement de cette discipline dans notre pays. Tous deux poursuivaient un même but, et ce malgré la concurrence qui régnait entre leurs cliniques, à savoir la reconnaissance de la chirurgie maxillo-faciale en tant que discipline spécialisée de la chirurgie basée sur une double formation. Lors de l'assemblée de fondation de la Société suisse de chirurgie maxillo-faciale (SSCMF) le 8 juin 1973 à Bâle, il a donc été ancré dans les statuts que la qualité

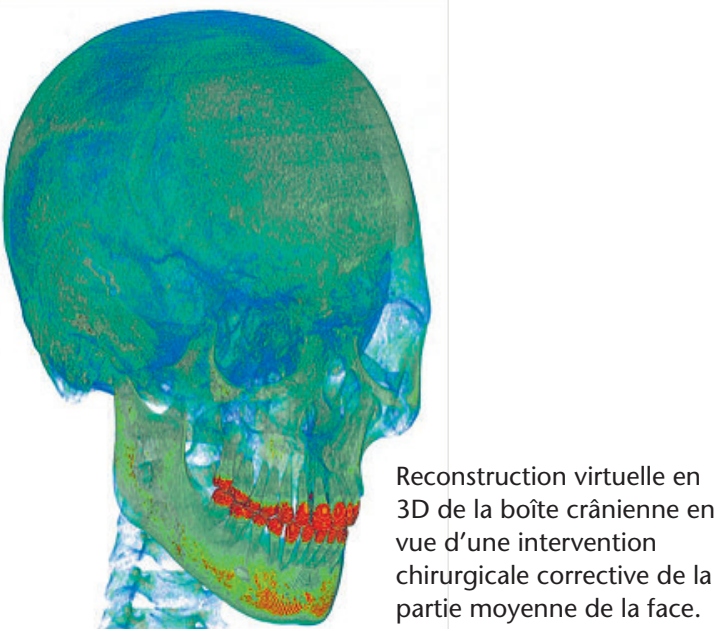

de membre ordinaire de la société ne pouvait s'acquérir qu'au bénéfice d'une double qualification.

\section{Turbulences autour du titre de spécialiste}

Des années ce sont toutefois écoulées jusqu'à la reconnaissance du titre par la FMH. Il a d'abord fallu balayer les oppositions de la Société suisse de chirurgie plastique et de la Société suisse d'oto-rhino-laryngologie, déjà membres de la FMH, qui exigeaient aussi la création d'un titre en chirurgie faciale. En juin 1984, le titre de «spécialiste en chirurgie maxillaire» a été créé, mais il a fallu attendre le début des années 90 pour que les craintes se dissipent et que le titre de spécialiste FMH en chirurgie maxillo-faciale soit approuvé.

Au sein de la société, il a aussi fallu créer les conditions nécessaires à l'élaboration et à l'acceptation d'une réglementation de la formation postgraduée permettant au médecin d'acquérir les compétences spécifiques nécessaires au traitement des structures maxillaires et faciales complexes. En Suisse, les candidats au titre suivent une formation préalable de deux ans en chirurgie générale avant d'accomplir quatre ans de formation clinique en chirurgie faciale.

La spécialisation du chirurgien maxillo-facial comprend la traumatologie, la chirurgie tumorale, le traitement des malformations congénitales et acquises du crâne facial, des malpositions maxillaires et des affections des articulations maxillaires, des infections du domaine maxillaire et facial, ainsi que des interventions de chirurgie orale et préprothétique.

\section{Garantir I'avenir}

Bernd Spiessl déclarait il y a une vingtaine d'années que ce n'est pas la double formation qu'il faut garantir, mais les bases appliquées de la chirurgie moderne ainsi que la qualité et l'avenir de la chirurgie maxillo-faciale. C'est sur cette remarque visionnaire que nous nous basons aujourd'hui encore pour assurer la pérennité d'un domaine exigeant marqué par les progrès rapides de la haute technologie (imagerie 3D et 4D, navigation, ingénierie tissulaire) et l'accroissement des possibilités thérapeutiques dans le domaine cervico-facial. En prévision des importants défis qui attendent la discipline, la SSCMF devra d'autant plus s'atteler à renforcer les synergies avec les sociétés apparentées telles que la société de neurochirurgie, d'oto-rhino-laryngologie, de chirurgie plastique ainsi que les sociétés de médecine dentaire et de mieux regrouper les compétences interdisciplinaires pour le bien du patient. 